\title{
The gut-bone axis: how bacterial metabolites bridge the distance
}

\author{
Mario M. Zaiss, ${ }^{1}$ Rheinallt M. Jones, ${ }^{2}$ Georg Schett, ${ }^{1}$ and Roberto Pacifici ${ }^{3,4}$ \\ 'Department of Internal Medicine 3, Rheumatology and Immunology, Friedrich-Alexander University Erlangen-Nürnberg (FAU) and Universitätsklinikum Erlangen, Erlangen, Germany. ²Department of \\ Pediatrics and ${ }^{3}$ Division of Endocrinology, Metabolism and Lipids, Department of Medicine, Emory University, Atlanta, Georgia, USA. ${ }^{4}$ Immunology and Molecular Pathogenesis Program, Emory University, \\ Atlanta, Georgia, USA.

\begin{abstract}
The gut microbiome is a key regulator of bone health that affects postnatal skeletal development and skeletal involution. Alterations in microbiota composition and host responses to the microbiota contribute to pathological bone loss, while changes in microbiota composition that prevent, or reverse, bone loss may be achieved by nutritional supplements with prebiotics and probiotics. One mechanism whereby microbes influence organs of the body is through the production of metabolites that diffuse from the gut into the systemic circulation. Recently, short-chain fatty acids (SCFAs), which are generated by fermentation of complex carbohydrates, have emerged as key regulatory metabolites produced by the gut microbiota. This Review will focus on the effects of SCFAs on the musculoskeletal system and discuss the mechanisms whereby SCFAs regulate bone cells.
\end{abstract}

\section{Introduction}

Osteoporosis is a common skeletal disease that leads to bone fractures and disability stemming from insufficient skeletal development leading to low peak bone density by age 30, and/or accelerated bone loss thereafter. Skeletal involution is determined by the process of bone remodeling, which involves the continuous removal of packets of old bone by the resorptive action of osteoclasts, and their replacement by new bone formed by osteoblasts $(1,2)$. Menopause, aging, inflammation, and hyperparathyroidism are common causes of osteoporosis that induce progressive loss of bone mineral density (BMD) by increasing osteoclastic bone resorption, or by decreasing osteoblast activity and lifespan.

Osteoporosis often remains untreated as a result of the cost and adverse side effects of approved drugs (3-6), underscoring the critical need for the development of inexpensive and safe interventions. To this end, recent investigations have focused on establishing the role of the gut microbiome in the development of osteoporosis, and in the efficacy of probiotics or prebiotics as novel approaches for its treatment. The notion that the gut microbiome is a BMD regulator in health and disease is supported by an established correlation in humans between microbiome diversity and osteoporosis (6). Moreover, animal studies have revealed that BMD is altered by the ablation of the gut microbiome, as it occurs in mice raised in germ-free (GF) conditions (7-9), and in mice treated with antibiotics (10-12).

Probiotics are defined as viable microorganisms that confer health benefits when administered in adequate quantities (13), while prebiotics are nondigestible fermentable food ingredients that promote the growth of beneficial microbes and/or promote

Conflict of interest: RP was the principal investigator in a research grant from VSL Pharmaceuticals.

Copyright: () 2019, American Society for Clinical Investigation.

Reference information: J Clin Invest. 2019;129(8):3018-3028.

https://doi.org/10.1172/JCI128521. beneficial changes in the activity of the microbiome (14). One mechanism whereby prebiotics and probiotics elicit positive health influences is by inducing modifications in the composition of the microbiota. These modifications, such as the expansion of Clostridia and Bacteroides, result in enhanced generation of metabolites that exert critical biological activities both in the gut and systemically. Indeed, metabolites produced in the gut by the microbiota provide an essential means for the gut microbiota to regulate anatomically distant organs. The term "postbiotics" is now used to refer to such metabolites. In addition to metabolites, structural components shed by bacteria may lead to distant effects on the organs of the body. For example, constituents of the bacterial cell wall such as peptidoglycan and lipopolysaccharide stimulate bone resorption (15).

\section{Gut-derived bacterial metabolites regulate distant organs}

The gut microbiome harbors hundreds of bacterial genera that reside in the luminal stream or adhere to the gut mucosa. The intestinal microbiome affords the host enhanced energy extraction from foodstuff, regulatory effects on epithelial growth, the exclusion of colonization by pathogens, and many other benefits (16). In addition, the gut microbiome is essential for efficient immune system maturation, as well as cytoprotection against exogenous insults. The gut microbiota produces metabolites that account for anatomically distant biological effects. Indole derivatives were among the first bacterial metabolites to be described to influence intestinal immunity $(17,18)$. In addition, trimethylamine $\mathrm{N}$-oxide (TMAO), an amine oxide present in food or generated within the human intestine by the microbiota from choline and carnitine, was found to negatively affect the vascular system and kidneys (19). 4-Ethylphenol sulfate, a metabolite produced by intestinal saprophytes, was shown to regulate human behavior and has been implicated in autism (20). Insulin-like growth factor 


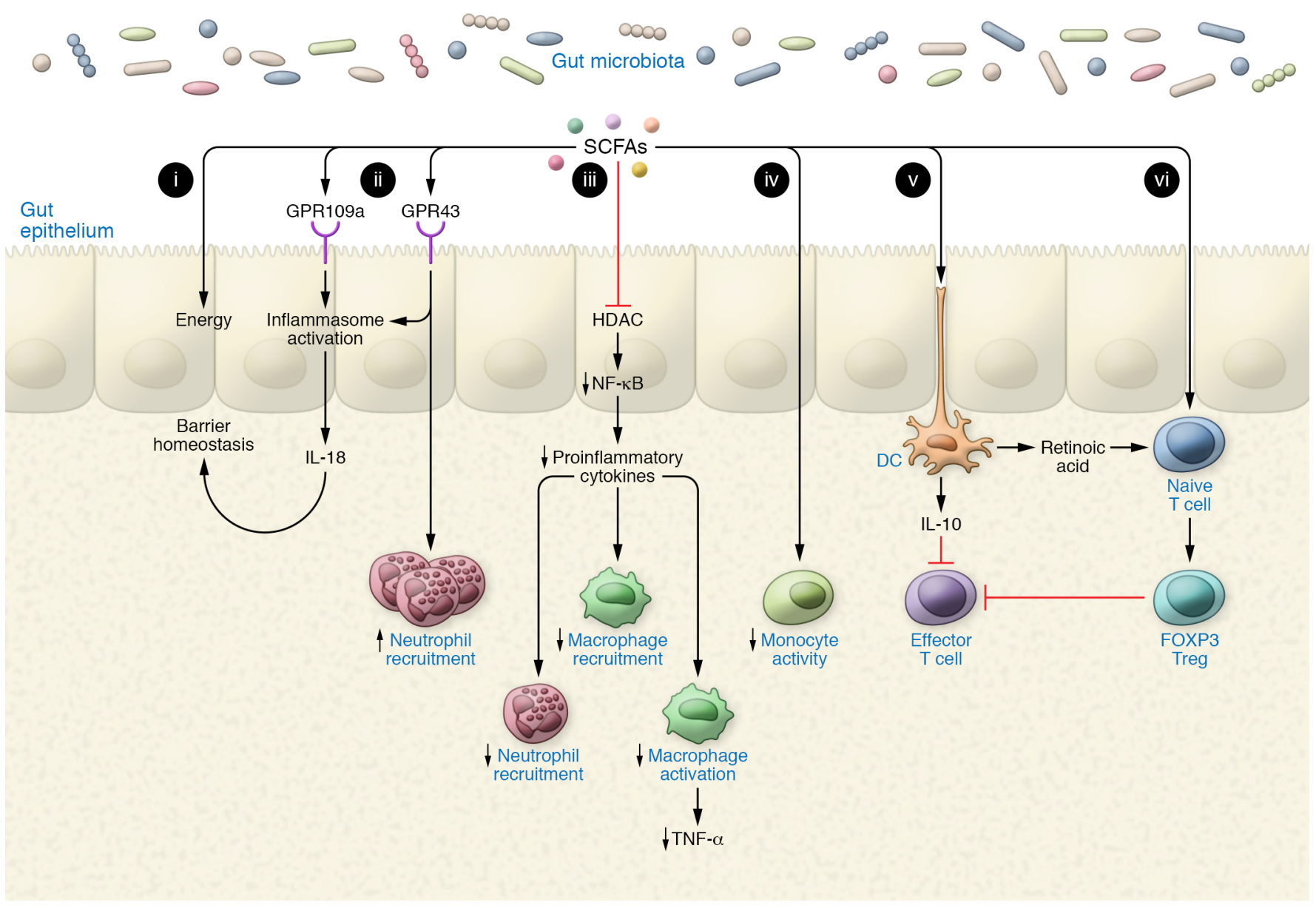

Figure 1. SCFAs bolster the gut epithelium and coerce a tolerogenic immune environment. (i) SCFAs act as a major and preferred energy source to the colonic epithelium. (ii) SCFAs signal via GPR43/109a to induce inflammasome activation, culminating in IL-18 secretion, which functions in gut barrier homeostasis. (iii) SCFAs dampen NF-kB signaling via HDAC inhibition, thereby inhibiting secretion of proinflammatory cytokines. (iv) SCFAs inhibit recruitment and activation of macrophages and neutrophils through a reduction in proinflammatory cytokine production. (v) SCFAs induce a tolerogenic dendritic cell phenotype by inducing the secretion of IL-10 and retinoic acid. IL-10 inhibits effector T cell function, while retinoic acid binds to the retinoic acid receptor in naive T cells to induce their differentiation into Tregs. (vi) SCFAs induce Treg differentiation through HDAC inhibition, which inhibits the activity of effector T cells, thus establishing a tolerogenic immune environment.

1 (IGF-1), produced predominantly in the liver in response to food intake and regulated by microbes and microbial products, was the first metabolite identified as a linker in the gut-bone axis $(8,21)$. Another bone-regulating molecule is hydrogen sulfide $\left(\mathrm{H}_{2} \mathrm{~S}\right)$, a gasotransmitter generated by gastrointestinal cells and by bacteria residing within the gut $(22,23)$. The microbiota accounts for a substantial portion of the overall blood levels of $\mathrm{H}_{2} \mathrm{~S}$, as GF mice have low serum and gastrointestinal tissue levels of $\mathrm{H}_{2} \mathrm{~S}(23)$. In turn, $\mathrm{H}_{2} \mathrm{~S}$ can modify the composition of the microbiota $(22,23)$. $\mathrm{H}_{2} \mathrm{~S}$ has been implicated in inflammatory bowel disease and other gastrointestinal pathological conditions. Importantly, $\mathrm{H}_{2} \mathrm{~S}$ stimulates bone formation and postnatal skeletal development (24). In addition, the $\mathrm{H}_{2} \mathrm{~S}$-donating compound GYY4137 increases bone formation by activating Wnt signaling via increased Wnt10b production (25), and prevents the loss of trabecular bone induced by ovariectomy (25). However, the family of metabolites produced by intestinal bacteria that has received the greatest attention for their capacity to diffuse to distant organs and induce potent regulatory effects are short-chain fatty acids (SCFAs). These metab- olites initially emerged as powerful immune cell controllers, and more recently have been recognized as pivotal regulators of bone resorption and bone formation.

\section{Sources and mechanisms of SCFA production}

Diet affects the diversity of the gut microbiota and thus by extension also influences the metabolic activity of the microbiome (26-28). A dietary element that plays a pivotal role in shaping the composition of the microbiome is vegetable fiber. This substance is regarded to be as essential as vitamins and other nutrients for organismal health. However, it is estimated that the current average consumption of fibers among adults in the United States is half the recommended amount of $30 \mathrm{~g}$ per day to be consumed as part of a healthy diet (29). Many of the beneficial health effects of fibers are due to metabolites generated by their digestion. Among these metabolites are SCFAs, which are derived from bacterial fermentation of complex nondigestible carbohydrates present in the diet. Amino acids and lactate catabolism also contribute to SCFA production, especially production of acetate and propionate $(30$, 


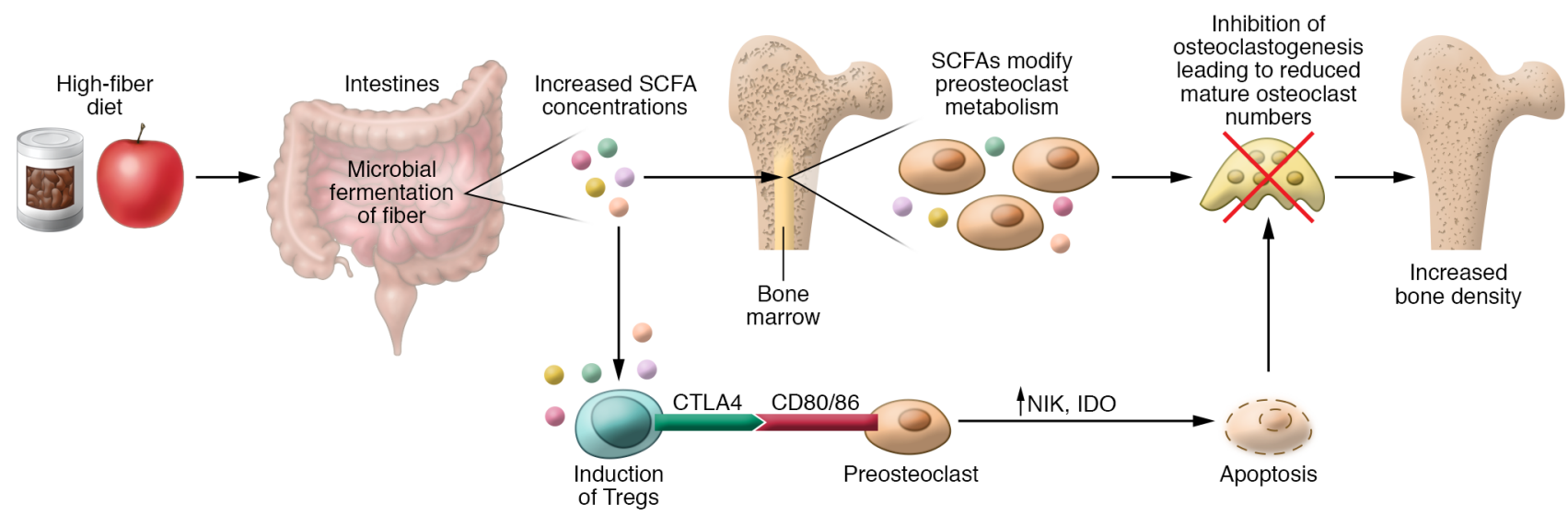

Figure 2. Direct and indirect effects of SCFAs on bone resorption. SCFAs, the main metabolites derived from microbial fermentation of dietary fibers in the intestine, affect bone homeostasis via two routes. In addition to butyrate's strong HDAC-inhibiting effects on osteoclasts, it directly induces metabolic reprogramming of osteoclast precursors, resulting in enhanced glycolysis at the expense of oxidative phosphorylation, thereby downregulating essential osteoclast genes such as TRAF6 and NFATc1. Indirect effects of SCFAs may account for their Treg-inducing capacity: Tregs were shown to suppress osteoclast differentiation via their secretion of antiosteoclastic cytokines as well as via a direct cell-cell contact-dependent, indoleamine 2,3-dioxygenaseinducing (IDO-inducing) mechanism. In summary, these data identify SCFAs as potent regulators of osteoclast metabolism and bone homeostasis.

31). Accordingly, individuals following a vegan or vegetarian diet, or those on a Mediterranean diet, have higher levels of SCFAs (32$45)$, and studies have indicated that these diets are beneficial to bone health $(46,47)$.

The SCFA generation pathways are well characterized and have been described in detail in review articles (48-50). The main SCFAs are butyrate, propionate, and acetate, which are found in the intestine in a molar ratio of 60:20:20, respectively (51). The human terminal ileum contains lower amounts of SCFAs (10 $\mathrm{mmol} / \mathrm{kg})$. In the cecum, concentrations are highest $(130 \mathrm{mmol} /$ $\mathrm{kg}$ ), while the distal colon contains intermediate concentrations of SCFAs $(80 \mathrm{mmol} / \mathrm{kg})(51)$. The concentrations of SCFAs in human blood are lower: e.g., $375 \mu \mathrm{mol} / \mathrm{L}$ in the portal blood, $150 \mu \mathrm{mol} / \mathrm{L}$ in the hepatic blood, and $80 \mu \mathrm{mol} / \mathrm{L}$ in the peripheral blood (12, 52). In mice, concentrations of SCFAs are quite variable, ranging from $0.1 \mathrm{mmol} / \mathrm{g}$ to $40 \mathrm{mmol} / \mathrm{g}$ in intestinal samples (53-60). The gut microbiota regulates the level of expression of enzymes involved in SCFA metabolism (61). The type and amount of SCFA produced by the gut microbiota also depends on the type of ingested nondigestible vegetable fibers, the duration of intestinal transit, and the composition and activity of the gut microbiota. Acetate is generated by many types of bacteria, while propionate and butyrate are only produced by a limited number of bacteria (62-64). For example, Akkermansia muciniphila generate propionic acid from the digestion of the mucus layer of the intestine (63). Butyrate is produced by few bacterial species in the microbiome, Faecalibacterium prausnitzii, Eubacterium rectale, Eubacterium hallii, and Ruminococcus bromii being representative examples (65). SCFAs are, first of all, an important source of energy, both for the microorganisms themselves and for the host. SCFAs provide approximately $10 \%$ of the energy requirement of humans consuming a Western-style diet (66). SCFAs are rapidly absorbed through the colonic mucosa, where butyrate is a critical source of energy for colonocytes. Propionate also provides energy to colonocytes, as well as to liver cells that utilize it for glucose formation, whereas acetate serves as a critical carbon source for lipid synthesis (67).

\section{SCFAs affect immune function and other biological systems}

SCFAs affect the immune system by modifying gene expression profiles $(68,69)$, cell chemotaxis $(70,71)$, differentiation $(54-56)$, proliferation (72-74), and apoptosis $(75,76)$. In addition, SCFAs inhibit histone deacetylases (HDACs) and stimulate histone acetyltransferase $(53,56,77,78)$ (Figure 1). Ligand binding to cell surface receptors or transmembrane transport by specialized transporters is critical for SCFA activities. SCFAs bind to four receptors, the free fatty acid receptors GPR 43 and GPR 41 (also known as FFAR2 and FFAR3) (79), the niacin/butyrate receptor GPR109a (also known as HCA2) (80), and the olfactory receptor Olfr78 (81). These receptors show distinct binding affinities for specific types of SCFAs, as well as different patterns of expression. An essential high-affinity SCFA transporter expressed in the intestine is Slc5a8. Accordingly, mice lacking Slc5a8 develop colitis and colon cancer (82), while GPR43 activation prevents colonic inflammation and carcinogenesis (83).

The role of SCFAs in the immune system has been extensively reviewed elsewhere $(53,71,84)$; some of the main discoveries are outlined herein. The intestinal epithelium acts as a barrier to prevent the passage of intraluminal entities such as foreign antigens, microorganisms, and their toxins into subepithelial compartments. Its second function is to act as a selective filter that allows the translocation of essential dietary nutrients, electrolytes, and water from the intestinal lumen into the circulation. Disrupted intestinal barrier function is associated with development of inflammatory diseases. One cause of impaired epithelial barrier integrity is low butyrate levels, which commonly occur as a result of microbiome dysbiosis (70). In addition, efficient intestinal barrier function can be restored upon supplementation of butyrate to the diet (85). Butyrate enhances intestinal barrier function by inducing the expression of tight junction proteins such as claudin-1 (4). Furthermore, the interaction of SCFAs with intestinal epithelial cells or immune cells of the gut mucosa leads to essential antiinflammatory and immunomodulatory effects. Among them is the enhanced production of antimicrobial peptides by intestinal mac- 


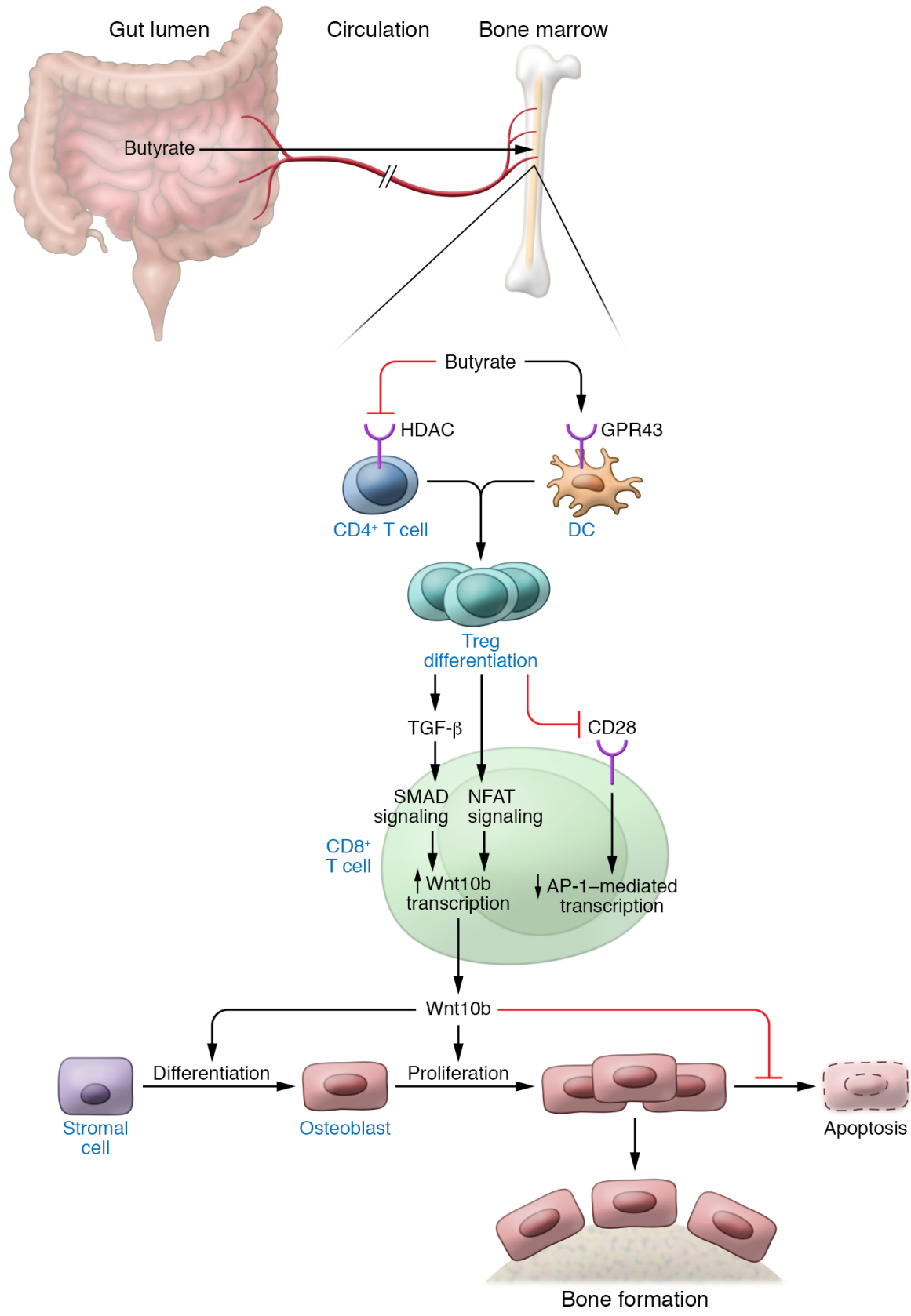

Figure 3. Lactobacillus rhamnosus GG and butyrate stimulate bone formation via a Treg-mediated mechanism. Dietary supplementation with the probiotic bacteria Lactobacillus rhamnosus CG (LCG) increases the relative frequency of Clostridia in the gut, which augments butyrate levels in intestinal tissue and serum. Butyrate enhances the differentiation of naive helper $\mathrm{CD}^{+}{ }^{+}$ cells into Tregs in the intestine, spleen, and bone marrow (BM). In the BM, Tregs block CD28 signaling in $C D 8^{+} T$ cells, thus dampening the nuclear levels of AP-1. In addition, Tregs activate NFAT and SMAD signaling, the latter via an increase in the production of TGF- $\beta$ by Tregs and conventional CD4 ${ }^{+} \mathrm{T}$ cells. Binding of NFAT and SMAD to the promoter region of Wnt10b (located between $-705 \mathrm{bp}$ and $-272 \mathrm{bp}$ ) potently activates Wnt10b expression. This osteogenic Wnt ligand activates Wnt signaling in BM stromal cells, causing their proliferation and differentiation into osteoblasts. The expansion of the osteoblastic population results in increased bone formation and improved bone structure.

The most studied immunomodulatory effect of SCFAs is their capacity to induce the differentiation and proliferation of regulatory T cells (Tregs) $(56,90,91)$. These cells are essential for the maintenance of immune tolerance and a symbiotic relationship between the host and the microbiome. Tregs suppress conventional $\mathrm{T}$ cells through a variety of mechanisms, including the production and secretion of the immunosuppressive cytokines IL-10 and TGF- $\beta(92,93)$. Several lines of evidence support the notion that SCFAs produced by the microbiome are critical for intestinal Treg expansion, including the observation that GF mice have fewer Tregs than conventionally raised mice, and reports that nutritional supplementation with SCFAs alone is sufficient to increase Treg numbers in the intestine of GF mice (56). Even modest alterations of the microbiota composition may lead to decreased pro-

rophages $(84,86)$, and NLRP3 inflammasome activation following SCFA receptor binding on intestinal epithelial cells (87). Additional effects of SCFAs on macrophages include the capacity to downregulate LPS-induced proinflammatory mediators such as nitric oxide, IL-6, and IL-12. These effects are independent of TLR signaling or SCFA receptor signaling but are rather due to inhibition of HDACs within immune cells by butyrate (88). Intestinal neutrophils are also regulated by SCFAs. Some of these effects, such as the enhancement of neutrophil migration via a GPR43-dependent mechanism, contribute to infection resolution in gut tissue (70), while others, like the inhibition of phagocytosis and blockade of the killing of Candida albicans, appear to be counterproductive (89). In these studies, SCFAs decreased the generation of IL-12, IFN- $\gamma$, and various chemokines, whereas SCFAs increased production of the antiinflammatory cytokine IL-10 $(69,83)$. duction of Tregs due to lower levels of SCFA generation, as attested by the finding of a paucity of Tregs in mice with dysbiosis as compared with mice with normal gut microbiota (94).

Butyrate and propionate are potent inducers of Tregs, whereas acetate modulates B cell function (95). In one study, propionate was more potent than acetate and butyrate, and was found to be sensed by Tregs via the fatty acid receptor GPR43 (56). Another report identified butyrate as the most potent inducer of Tregs (91). Interestingly, oral administration of SCFAs following antibiotic treatment to mice showed that a combination of propionate and butyrate expands the Treg population in the spleen, whereas a combination of propionate and acetate expands Treg numbers in the intestine (90).

SCFAs support the development of peripheral Tregs in the intestine through multiple mechanisms. A key effect of SCFAs is to increase the differentiation of naive $\mathrm{CD} 4^{+} \mathrm{T}$ cells into Tregs 
via intrinsic epigenetic upregulation of the Foxp3 gene in T cells. This effect, which is surface receptor-independent (87), is mediated by increased histone $\mathrm{H} 3$ acetylation of the promoter for $\mathrm{Cns} 3$ and Cns1 gene loci (91). Another effect of SCFAs is to increase the proliferation of mature Tregs (56). This effect was reported to be GPR43-dependent in one study (56) and GPR43-independent in another study (91). Additional effects of SCFAs, especially butyrate, on Treg maturation are mediated by dendritic cells (DCs) (90), which express both GPR109a and GPR43 (56, 83). Since GPR43 is expressed at high levels by myeloid cells (91), it is likely that GPR43 mediates the effects of SCFAs on DCs. Butyrate and propionate (but not acetate) prevent the development of DCs via inhibition of histone acetyltransferase (82). Butyrate prevents DC maturation by upregulating antiinflammatory genes (96). This effect results in an increased capacity of DCs to support Treg differentiation. Butyrate and propionate induce DCs to promote the formation of IL-10-producing Tregs and inhibit the generation of IFN- $\gamma^{+}$effector T cells (83). Altogether, these reports highlight the sensitive responses of immune cells within gut mucosa and beyond to varying concentrations of SCFAs.

\section{Effects of SCFAs on osteoclasts and bone resorption}

Osteoclasts are multinucleated cells that are responsible for physiological and pathological bone resorption. SCFAs blunt osteoclast differentiation (97), and inhibition of HDAC activity is one mechanism whereby this occurs (98-100). For example, differentiation of primary bone marrow (BM) cells into osteoclasts is suppressed by butyrate and by trichostatin A, a known HDAC inhibitor (101). Treatment with the newer HDAC inhibitor depsipeptide FR901228 confirmed the antiosteoclastic properties of butyrate, suggesting a novel role for HDAC inhibitors as antiresorptive agents (102). Two further studies highlighted the antiosteoclastic effects of butyrate, and to a lesser extent propionate, on osteoclast differentiation using mice and human primary cultured cells $(103,104)$. Suppression of osteoclast differentiation is most potent when SCFAs or HDAC inhibitors are added at early time points during osteoclast differentiation $(53,55,101)$.

Mice lacking FFR1 (GPR40), a receptor that binds mid- to long-chain fatty acids, were protected against bone loss through suppression of osteoclastogenesis (105). This report led to further investigations by our laboratory on the potential of fiber-rich diets (prebiotic), bacterial transfer (probiotic), or nutritional supplementation with SCFAs (postbiotic) on bone metabolism under steady-state and bone waste-inducing conditions. We reported beneficial effects of all three approaches on bone metabolism (55). We also showed reduced osteoclast numbers in C57BL/6 mice and in osteoporotic mice following propionate and butyrate treatment (55). These observations correlated with significantly reduced markers for bone resorption.

Earlier publications highlighted the potential of Tregs to attenuate osteoporosis (106), and to increase systemic BMD by directly suppressing osteoclast differentiation (107-109) in a CTLA4/ CD80/86 cell-cell contact-inducing and indoleamine 2,3-dioxygenase-inducing manner (110) (Figure 2). Considering the extensive body of literature reporting on the Treg-inducing potential of SCFAs $(56,90,94)$, it was unexpected to find increased systemic bone densities in Rag1-/ ( $\mathrm{T}$ and B cell-deficient) mice following propionate and butyrate treatment. The direct osteoclast-suppressing effects of propionate and butyrate - contrary to the indirect mechanism via expansion of Tregs - were shown to be independent of the receptors GPR41 and GPR43, and rather occurred via a shift in cellular metabolism toward increased glycolysis at early time points during osteoclast differentiation. Blocking glycolysis during this time window rescued the anti-osteoclastogenic potential of propionate and butyrate in in vitro experiments (55). To test whether these findings could be exploited as a novel strategy for the treatment of pathological bone loss, we further investigated the impact of SCFAs on ovariectomy-induced bone loss. In line with the capacity of propionate and butyrate to induce metabolic shifts toward increased glycolysis in osteoclast precursors, these SCFAs effectively prevented ovariectomy-induced bone loss. Moreover, propionate and, even more potently, butyrate prevented the ovariectomy-induced increase in osteoclast formation and bone resorption. By contrast, markers of bone formation remained unchanged. Interestingly, while high-fiber diets in steady-state wild-type mice increased bone mass, no positive effects on bone volume were observed when ovariectomized mice were fed a high-fiber diet (55). Studies are in progress to confirm the anti-osteoclastogenic activity of SCFAs in humans.

Accumulated research has revealed that the source, concentration, and amino acid balance of dietary protein are additional factors that positively contribute to the composition, structure, and function of the gut microbiome. Therefore, in a first-inhuman trial registered in the German Clinical Trials Register (DRKS00017277), we combined protein supplementation with a high-fiber diet, and observed enhanced production of Tregs and decreased bone resorption in subjects receiving supplements. This dietary combination strongly improved the acceptance and willingness of patients to consume the protein supplementation. Thus, increases in SCFA levels that occur as a result of pre-, pro-, or postbiotic dietary supplementation may serve as an inexpensive, safe, and effective intervention for both the prevention and treatment of osteoporosis.

\section{Effects of SCFAs on osteoblasts and bone formation}

Physiological stimuli and pharmacological agents that increase bone formation typically act by increasing osteoblastogenesis, increasing osteoblast lifespan, or a combination of both. Activation of Wnt signaling in osteoblasts plays an essential role in increasing osteoblastogenesis and decreasing osteoblast apoptosis $(111,112)$. Wnt signaling is indeed critical for bone mass acquisition and skeletal involution (113). Attesting to the relevance of Wnt signaling for skeletal health and disease, activating mutations of the Wnt signaling coreceptor complex result in a high-bone mass phenotype (114), while inactivating mutations are responsible for low bone mass and the early onset of osteoporosis (115). Activation of the canonical Wnt signaling pathway results from increased production of Wnt ligands that bind to and activate the Wnt coreceptor complex, or from diminished production of Wnt signaling inhibitors such as Dkk1 (116) and sclerostin (117). Emerging reports have described skeletal effect of SCFAs, including investigations showing that butyrate promotes the osteogen- 
ic differentiation of stromal cells (118), and mineralized nodule formation (119). Moreover, dietary supplementation with oligosaccharides that increase SCFA generation also increased BMD (120). On the other hand, SCFAs supplementation is reported to decrease bone volume without altering bone turnover rates in mice treated with antibiotics (21). These discoveries prompted a need to examine the effects of SCFAs on bone volume in mice with normal gut microbiota (Figure 3).

Tregs are suppressive $\mathrm{CD}^{+} \mathrm{T}$ cells that reside preferentially on the endosteal surfaces of bone (121); they are capable of suppressing osteoclastogenesis (107-109) and promoting osteoblast differentiation $(122,123)$ and are required for parathyroid hormone-stimulated (PTH-stimulated) bone formation (124). The fact that SCFAs promote the differentiation of naive $\mathrm{CD} 4^{+}$cells into Tregs $(56,90$, 91) suggests that SCFAs and probiotics that increase the production of SCFAs, such as Lactobacillus rhamnosus GG (LGG), may act through a pathway linking SCFAs, Tregs, and bone formation. This notion was the subject of a recent report from our laboratory in which we showed that dietary supplementation with LGG for 4 weeks altered the composition of the intestinal microbiota (60). The most relevant change was an increase in the number of Clostridia, which are known to induce the production of SCFAs. Indeed, LGG treatment increased intestinal and circulating butyrate, a finding confirming the capacity of butyrate to diffuse from the intestine to distant organs. Butyrate and LGG were equally capable of stimulating bone formation and increasing trabecular bone volume without affecting cortical bone. This is surprising for several reasons: First, the data provide robust evidence that LGG, and probably all lactobacilli-containing probiotics, are capable of favorably altering postnatal skeletal development in young animals. This has not been consistently observed in previous studies with other probiotics (35). Second, the data indicate that the skeletal effects of LGG in eugonadic mice are mediated by butyrate, raising the possibility that butyrate and perhaps other SCFAs may represent a novel therapeutic approach for osteoporosis or optimization of skeletal development in children. Third, the result of this investigation and a previous study in ovariectomized mice (36) provide evidence that LGG exerts skeletal effects through multiple mechanisms that are dependent on the physiological status of the host. In contrast to eugonadic mice, in which the prevailing regulatory event is metabolic activity mediated by SCFAs (60), in sex steroid-deficient mice, LGG exerts a bone-sparing effect due to a positive modulatory effect on gut permeability and gut inflammation (36). These differences may be related to the fact that sex steroid-deficient mice have a higher rate of bone turnover, a higher inflammatory state, and increased gut permeability as compared with eugonadic mice. Fourth, it should be underlined that in both sex steroid-deficient mice and eugonadic mice, the positive skeletal effects of LGG were limited to the trabecular compartment. These findings underscore the fact that the cortical envelope of the skeleton is regulated differently compared with the trabecular compartment.

Strong experimental evidence supports the notion that the capacity of butyrate to stimulate bone formation is due to an increase in the number of Tregs in the BM. In fact, studies where the expansion of Tregs was prevented by injection of anti-CD25 antibody revealed that butyrate is unable to induce bone formation and increase bone mass if Tregs are absent (60). This was confirmed using DEREG mice, a knockin strain expressing the human diphtheria receptor in Tregs. Treatment of DEREG mice with diphtheria toxin causes the ablation of Tregs. Likewise, butyrate is unable to induce bone formation and increase bone mass in DEREG mice treated with a dose of diphtheria toxin. Since there was no evidence of increased inflammation, these experiments excluded the possibility that Treg depletion blocked the bone anabolic activity of butyrate by inducing inflammation. Partial Treg blockade also prevented the increase in Wnt10b production by $\mathrm{CD} 8^{+} \mathrm{T}$ cells induced by butyrate. This finding is noteworthy because Wnt10b is a key activator of Wnt signaling in stromal cells and osteoblasts. Wnt10b increases osteoblast proliferation (115), differentiation $(125,126)$, and survival (127-129), and regulates the production of osteoprotegerin (130). In humans, Wnt10b is a predictor of bone mass (131), while in mice Wnt10b is essential for bone mass acquisition at baseline conditions $(132,133)$, and its deficiency results in age-dependent bone loss (134). The function of Wnt10b as an endogenous Wnt ligand operating in bone is further supported by the observation that heterozygous $W n t 10 b^{+/-}$ mice exhibit a significant reduction of trabecular bone (134). Moreover, the probiotic Lactobacillus reuteri prevents diabetes-induced bone loss by upregulating Wnt10b (135), while the specific pool of Wnt10b produced by $\mathrm{CD}^{+} \mathrm{T}$ cells is a critical inducer of bone formation in response to PTH (136-138).

The discovery that an increase in the number of Tregs in the $\mathrm{BM}$ affects the expression of Wnt10b by $\mathrm{CD}^{+} \mathrm{T}$ cells raises the question of the involved mechanism. The Wnt10b gene promoter region harbors three DNA-binding motifs for NFAT transcription factors located adjacent to binding sites for SMADs, the TGF- $\beta$ signaling proteins. This organization suggests that Wnt10b transcription may be regulated by the binding of NFAT/SMAD dimers to the $W n t 10 b$ promoter. Indeed, one of these binding sites, located between $-705 \mathrm{bp}$ and $-272 \mathrm{bp}$ in the $W n+10 b$ promoter, was found to be critical for Wnt10b transcription induced by LGG or butyrate (60). In the context of $\mathrm{T}$ cell activation, the preferred partner of NFAT is AP-1, not SMADs $(139,140)$. By silencing CD28 signaling in $\mathrm{CD}^{+} \mathrm{T}$ cells, Tregs lower the production of AP- 1 and favor the binding of NFAT to SMADs (141). Accordingly, butyrate increased the binding of NFAT1 and SMAD3 to the Wnt1Ob promoter, but only when the number of Tregs was increased. In summary, Tregs promote the assembly of an NFAT1-SMAD3 transcription complex in $\mathrm{CD}^{+}$cells, which drives the expression of Wnt10b.

\section{SCFAs and PTH: mechanistic similarities and evolutionary considerations}

PTH is a calciotropic hormone critical for skeletal development. Similarly to butyrate, PTH stimulates bone formation and induces bone anabolism via the Treg/Wnt10b/Wnt signaling pathway $(125,142,143)$. BM CD8 ${ }^{+} \mathrm{T}$ cells respond to PTH and butyrate by releasing Wnt10b, while silencing of Wnt10b expression by $\mathrm{CD} 8^{+}$ T cells blocks the capacity of PTH and butyrate to stimulate bone formation and increase bone volume (60, 136-138). Moreover, PTH and butyrate increase the production of Wnt10b by $\mathrm{CD}^{+} \mathrm{T}$ cells by expanding Tregs $(60,124)$.

The evolutionary advantage of the mechanistic convergence between the skeletal effects of SCFAs and those of PTH remains unknown, but it is tempting to speculate that it may be related to 
energy balance during health and sickness. Immune cells depend on calcium for their activation (144). A highly activated immune system is accompanied by sickness behavior and anorexia, which renders the immune system dependent on calcium released by bone resorption rather than the calcium absorbed in the gut. A consequence of starvation is hypocalcemia that in turn leads to continuous production of PTH, which stimulates bone resorption, causing release of calcium, which then becomes available for immune cell activation (145). Food ingestion interrupts PTH secretion, causing the pattern of PTH release to change from continuous to intermittent. It is only when intermittently produced that PTH exerts a net bone anabolic activity. This activity hinges on a mechanism involving Tregs and Wnt1Ob. One goal of this response might be to induce calcium deposition in the skeleton, so as to create a calcium reserve for the immune system. Generation of SCFAs is an event linked to food intake. Thus, SCFA generation may signal the presence of a normal state of health, thereby activating a pathway that replenishes calcium reserve in the skeleton. Thus, SCFAs may act in concert with intermittent PTH release to expand Tregs and stimulate bone formation.

\section{Modulation of gut-bone axis by probiotics and prebiotics}

SCFA supplementation is emerging as a novel postbiotic treatment modality for optimizing postnatal skeletal development and preventing pathological bone loss. Pre- and probiotics also act, in part, by generating SCFAs that positively affect the skeleton (60, 146-148). In addition, these interventions suppress inflammation (149), regulate the immune responses in the host $(41,150)$, buttress a weak gut epithelial barrier $(151,152)$, and promote epithelial development and restitutional responses following injury (153-155). Robust evidence demonstrates that probiotics prevent the bone loss induced by ovariectomy, a model of postmenopausal osteoporosis $(32-34,36)$, prevent the bone loss induced by periodontal disease (156) and diabetes (135), and are beneficial for skeletal health in intact animals (35, 60, 157-159). Moreover, increasing evidence indicates that probiotics positively affect skeletal health in humans. Early trials showed that ingestion of kefir fermented milk for 6 months caused an increase in BMD in men (45), while treatment with Lactobacillus casei shirota improved distal radius fracture healing in elderly men and women (160). Another trial with a multispecies probiotic showed a significant reduction in bone turnover, but no significant changes in BMD, perhaps because the trial duration was only 6 months (42). A 1-year-long trial in older women revealed evidence of a favorable change in bone mass in response to probiotic supplementation (43), and in a study in Japanese women, the probiotic Bacillus subtilis C-3102 increased total hip bone BMD by decreasing bone resorption (44).

Prebiotics, which are predominantly nondigestible substances that act as food for the gut microbiota, are found in a variety of foodstuffs, such as artichoke, garlic, leek, dandelion greens, banana, onion, and chicory (161). Prebiotics include nondigestible oligosaccharides and soybean oligosaccharides. In many cases, a substantial amount of the food must be consumed to acquire enough prebiotic for activity, and therefore prebiotics, such as inulin, have been developed into soft chew, capsule, tablet, and shake forms (161). Prebiotics prevent ovariectomy-induced bone loss in rats (162) and increase BMD in healthy animals $(37,38,163)$. In humans, prebiotics increase BMD in adolescents (164) and decrease bone turnover in postmenopausal women (165). The mechanism of action of prebiotics in bone is complex, but emerging evidence has shown that bacterial metabolic pathways, including those that function in the generation of SCFAs, are involved (148).

\section{Conclusions and future directions}

SCFAs exert complex effects in bone remodeling that suggest novel therapeutic opportunities for SCFAs in the treatment of metabolic bone disorders. In addition, nutritional supplementation with prebiotics and probiotics that increase SCFA production may represent an effective, safe, and inexpensive modality to prevent and treat osteoporosis. Additional studies will be required to identify the pre- and probiotic formulations that maximize SCFA production. While current efforts are focused on the identification of bacterial strains that provide maximal skeletal effects, it is entirely possible that the number of bacteria surviving passage through gastric acid, rather than bacterial species, will emerge as a key factor for probiotic efficacy. In animal models SCFAs have been shown to blunt osteoclastogenesis and bone resorption and stimulate bone formation. The antiresorptive activity of SCFAs is T cell-independent (55), while the bone anabolic activity of SCFAs is dependent on Tregs and CD8 ${ }^{+} \mathrm{T}$ cells (60). The factors that determine whether SCFAs act primarily as antiresorptive agents or as anabolic agents are unknown. However, the composition of the microbiota, the source and age of the treated mice, and the duration of the treatment are certainly relevant factors, highlighting the fact that it is essential to account for reciprocal host-microbiome interactions in experimental science. Efforts to understand the factors that determine the bone cell response to SCFAs will be an important subject for future research. Most of the evidence linking microbiota-produced metabolites to bone derives from animal studies. It will be critical to confirm these observations in humans and thereafter conduct clinical trials with emerging postbiotic agents.

It is estimated that metabolites of bacterial origin account for about $10 \%$ of circulating metabolites (166). We predict that rapid progress in metabolomic and other emerging technologies will lead to the discovery of several metabolites critical for the regulation of bone turnover and the maintenance of bone health. Novel immune-metabolic pathways are likely to be identified that will provide innovative therapeutic opportunities for metabolic bone diseases.

\section{Acknowledgments}

This study was supported by grants from the NIH (DK1129461, DK108842, DK119229, and RR028009 to RP; DK098391 to RMJ). MZ was supported by the Else Kröner-Fresenius-Stiftung, the Johannes und Frieda Marohn-Stiftung, and the ELAN program of the Interdisciplinary Center for Clinical Research (IZKF) at the Friedrich-Alexander University Erlangen-Nürnberg. MZ and GS were supported by DFG-FOR2886 PANDORA-A01 and DFGCRC1181-B07 from the Deutsche Forschungsgemeinschaft (German Research Foundation), and the ERC Synergy Grant 4D Nanoscope (to GS). The Emory Gnotobiotic Animal Core is supported by the Georgia Clinical and Translational Science Alliance and the 
Emory University School of Medicine. The Emory Integrated Core Facilities are supported by the Emory Neuroscience NINDS Core Facilities, the Georgia Clinical and Translational Science Alliance, and the Emory University School of Medicine.
Address correspondence to: Roberto Pacifici, Division of Endocrinology, Metabolism and Lipids, Emory University School of Medicine, 101 Woodruff Circle, Room 1309, Atlanta, Georgia 30322, USA. Phone: 404.712.8420; Email: roberto.pacifici@emory.edu.
1. Feng X, McDonald JM. Disorders of bone remodeling. Annu Rev Pathol. 2011;6:121-145.

2. Parfitt AM. Targeted and nontargeted bone remodeling: relationship to basic multicellular unit origination and progression. Bone. 2002;30(1):5-7.

3. Khosla S, Shane E. A crisis in the treatment of osteoporosis. JBone Miner Res. 2016;31(8):1485-1487.

4. Jha S, Wang Z, Laucis N, Bhattacharyya T. Trends in media reports, oral bisphosphonate prescriptions, and hip fractures 1996-2012: an ecological analysis. J Bone Miner Res. 2015;30(12):2179-2187.

5. Kim SC, et al. Impact of the U.S. food and drug administration's safety-related announcements on the use of bisphosphonates after hip fracture. J Bone Miner Res. 2016;31(8):1536-1540.

6. Wang J, et al. Diversity analysis of gut microbiota in osteoporosis and osteopenia patients. PeerJ. 2017;5:e3450.

7. Sjögren K, et al. The gut microbiota regulates bone mass in mice. J Bone Miner Res. 2012;27(6):1357-1367.

8. Novince $\mathrm{CM}$, et al. Commensal gut microbiota immunomodulatory actions in bone marrow and liver have catabolic effects on skeletal homeostasis in health. Sci Rep. 2017;7(1):5747.

9. Blanton LV, et al. Gut bacteria that prevent growth impairments transmitted by microbiota from malnourished children. Science. 2016;351(6275):aad3311.

10. Cho I, et al. Antibiotics in early life alter the murine colonic microbiome and adiposity. Nature. 2012;488(7413):621-626.

11. Pytlik M, Folwarczna J, Janiec W. Effects of doxycycline on mechanical properties of bones in rats with ovariectomy-induced osteopenia. Calcif Tissue Int. 2004;75(3):225-230.

12. Cox LM, et al. Altering the intestinal microbiota during a critical developmental window has lasting metabolic consequences. Cell. 2014;158(4):705-721.

13. Pintado J, Guyot JP, Ampe F. Multiple competitive PCR-DGGE as a tool for quantifying and profiling defined mixed cultures of lactic acid bacteria during production of probiotics from complex polysaccharides. J Appl Microbiol. 2003;95(5):921-933.

14. Gibson GR, Probert HM, Loo JV, Rastall RA, Roberfroid MB. Dietary modulation of the human colonic microbiota: updating the concept of prebiotics. Nutr Res Rev. 2004;17(2):259-275.

15. Kishimoto T, et al. Peptidoglycan and lipopolysaccharide synergistically enhance bone resorption and osteoclastogenesis. J Periodont Res. 2012;47(4):446-454

16. Blaser MJ. The microbiome revolution. JClin Invest. 2014;124(10):4162-4165.

17. Zelante T, et al. Tryptophan catabolites from microbiota engage aryl hydrocarbon receptor and balance mucosal reactivity via interleukin-22. Immunity. 2013;39(2):372-385.
18. Schiering C, et al. Feedback control of AHR signalling regulates intestinal immunity. Nature. 2017;542(7640):242-245.

19. Kelly CJ, et al. Crosstalk between microbiota-derived short-chain fatty acids and intestinal epithelial HIF augments tissue barrier function. Cell Host Microbe. 2015;17(5):662-671.

20. Hsiao EY, et al. Microbiota modulate behavioral and physiological abnormalities associated with neurodevelopmental disorders. Cell. 2013;155(7):1451-1463.

21. Yan J, et al. Gut microbiota induce IGF-1 and promote bone formation and growth. Proc Natl Acad Sci U S A. 2016;113(47):E7554-E7563.

22. Linden DR. Hydrogen sulfide signaling in the gastrointestinal tract. Antioxid Redox Signal. 2014;20(5):818-830.

23. Shen X, Carlström M, Borniquel S, Jädert C, Kevil CG, Lundberg JO. Microbial regulation of host hydrogen sulfide bioavailability and metabolism. Free Radic Biol Med. 2013;60:195-200.

24. Liu Y, et al. Hydrogen sulfide maintains mesenchymal stem cell function and bone homeostasis via regulation of $\mathrm{Ca}(2+)$ channel sulfhydration. Cell Stem Cell. 2014;15(1):66-78.

25. Grassi F, et al. Hydrogen sulfide is a novel regulator of bone formation implicated in the bone loss induced by estrogen deficiency. J Bone Miner Res. 2016;31(5):949-963.

26. Brüssow H, Parkinson SJ. You are what you eat. Nat Biotechnol. 2014;32(3):243-245.

27. Louis P, Scott KP, Duncan SH, Flint HJ. Understanding the effects of diet on bacterial metabolism in the large intestine. J Appl Microbiol. 2007;102(5):1197-1208.

28. Clemente JC, Ursell LK, Parfrey LW, Knight $\mathrm{R}$. The impact of the gut microbiota on human health: an integrative view. Cell. 2012;148(6):1258-1270.

29. Millen BE, et al. The 2015 Dietary Guidelines Advisory Committee Scientific Report: development and major conclusions. Adv Nutr. 2016; 7(3):438-444.

30. Bourriaud C, et al. Lactate is mainly fermented to butyrate by human intestinal microfloras but inter-individual variation is evident. J Appl Microbiol. 2005;99(1):201-212.

31. Neis EP, Dejong CH, Rensen SS. The role of microbial amino acid metabolism in host metabolism. Nutrients. 2015;7(4):2930-2946.

32. Chiang SS, Pan TM. Antiosteoporotic effects of Lactobacillus -fermented soy skim milk on bone mineral density and the microstructure of femoral bone in ovariectomized mice. J Agric Food Chem. 2011;59(14):7734-7742.

33. Ohlsson C, et al. Probiotics protect mice from ovariectomy-induced cortical bone loss. PLoS One. 2014;9(3):e92368.

34. Britton RA, et al. Probiotic L. reuteri treatment prevents bone loss in a menopausal ovariectomized mouse model.JCell Physiol.
2014;229(11):1822-1830

35. Collins FL, et al. Lactobacillus reuteri 6475 increases bone density in intact females only under an inflammatory setting. PLoS One. 2016;11(4):e0153180.

36. Li JY, et al. Sex steroid deficiency-associated bone loss is microbiota dependent and prevented by probiotics. JClin Invest. 2016;126(6):2049-2063.

37. Roberfroid MB, Cumps J, Devogelaer JP. Dietary chicory inulin increases whole-body bone mineral density in growing male rats. J Nutr. 2002;132(12):3599-3602

38. Takahara S, Morohashi T, Sano T, Ohta A, Yamada S, Sasa R. Fructooligosaccharide consumption enhances femoral bone volume and mineral concentrations in rats. J Nutr. 2000;130(7):1792-1795.

39. Scholz-Ahrens KE, Schrezenmeir J. Inulin and oligofructose and mineral metabolism: the evidence from animal trials. J Nutr. 2007; 137(11 suppl):2513S-2523S.

40. Scholz-Ahrens KE, et al. Glucocorticosteroid-induced osteoporosis in adult primiparous Göttingen miniature pigs: effects on bone mineral and mineral metabolism. Am J Physiol Endocrinol Metab. 2007;293(1):E385-E395.

41. Scholz-Ahrens KE, et al. Prebiotics, probiotics, and synbiotics affect mineral absorption, bone mineral content, and bone structure. J Nutr. 2007;137(3 suppl 2):838S-846S.

42. Jafarnejad S, Djafarian K, Fazeli MR, Yekaninejad MS, Rostamian A, Keshavarz SA. Effects of a multispecies probiotic supplement on bone health in osteopenic postmenopausal women: a randomized, double-blind, controlled trial. J Am Coll Nutr. 2017;36(7):497-506.

43. Nilsson AG, Sundh D, Bäckhed F, Lorentzon M. Lactobacillus reuteri reduces bone loss in older women with low bone mineral density: a randomized, placebo-controlled, double-blind, clinical trial. J Intern Med. 2018;284(3):307-317.

44. Takimoto T, et al. Effect of Bacillus subtilis C-3102 on bone mineral density in healthy postmenopausal Japanese women: a randomized, placebo-controlled, double-blind clinical trial. Biosci Microbiota Food Health. 2018;37(4):87-96.

45. Tu MY, Chen HL, Tung YT, Kao CC, Hu FC, Chen CM. Short-term effects of kefir-fermented milk consumption on bone mineral density and bone metabolism in a randomized clinical trial of osteoporotic patients. PLoS One. 2015;10(12):e0144231.

46. Marsh AG, Sanchez TV, Midkelsen O, Keiser J, Mayor G. Cortical bone density of adult lacto-ovo-vegetarian and omnivorous women. JAm Diet Assoc. 1980;76(2):148-151.

47. Rivas A, et al. Mediterranean diet and bone mineral density in two age groups of women. Int $J$ Food Sci Nutr. 2013;64(2):155-161.

48. Flint HJ, Duncan SH, Scott KP, Louis P. Links between diet, gut microbiota composition and gut metabolism. Proc Nutr Soc. 2015;74(1):13-22. 
49. Miller TL, Wolin MJ. Pathways of acetate, propionate, and butyrate formation by the human fecal microbial flora. Appl Environ Microbiol. 1996;62(5):1589-1592.

50. Koh A, De Vadder F, Kovatcheva-Datchary P, Bäckhed F. From dietary fiber to host physiology: short-chain fatty acids as key bacterial metabolites. Cell. 2016;165(6):1332-1345.

51. Cummings JH, Pomare EW, Branch WJ, Naylor CP, Macfarlane GT. Short chain fatty acids in human large intestine, portal, hepatic and venous blood. Gut. 1987;28(10):1221-1227.

52. Pomare EW, Branch WJ, Cummings JH. Carbohydrate fermentation in the human colon and its relation to acetate concentrations in venous blood. J Clin Invest. 1985;75(5):1448-1454.

53. Fellows R, et al. Microbiota derived short chain fatty acids promote histone crotonylation in the colon through histone deacetylases. Nat Commun. 2018;9(1):105.

54. Haghikia A, et al. Dietary fatty acids directly impact central nervous system autoimmunity via the small intestine. Immunity. 2015;43(4):817-829.

55 . Lucas S, et al. Short-chain fatty acids regulate systemic bone mass and protect from pathological bone loss. Nat Commun. 2018;9(1):55.

56. Smith PM, et al. The microbial metabolites, short-chain fatty acids, regulate colonic Treg cell homeostasis. Science. 2013;341(6145):569-573.

57. Tahara Y, et al. Gut microbiota-derived short chain fatty acids induce circadian clock entrainment in mouse peripheral tissue. Sci Rep. 2018;8(1):1395.

58. Trompette A, et al. Gut microbiota metabolism of dietary fiber influences allergic airway disease and hematopoiesis. Nat Med. 2014;20(2):159-166.

59. Zaiss MM, et al. The intestinal microbiota contributes to the ability of helminths to modulate allergic inflammation. Immunity. 2015;43(5):998-1010.

60. Tyagi AM, et al. The microbial metabolite butyrate stimulates bone formation via $\mathrm{T}$ regulatory cell-mediated regulation of WNT10B expression. Immunity. 2018;49(6):1116-1131.e7.

61. Cherbuy C, et al. Expression of mitochondrial HMGCoA synthase and glutaminase in the colonic mucosa is modulated by bacterial species. Eur J Biochem. 2004;271(1):87-95.

62. Morrison DJ, Preston T. Formation of short chain fatty acids by the gut microbiota and their impact on human metabolism. Gut Microbes. 2016;7(3):189-200.

63. Derrien M, Vaughan EE, Plugge CM, de Vos WM. Akkermansia muciniphila gen. nov., sp. nov., a human intestinal mucin-degrading bacterium. Int J Syst Evol Microbiol. 2004;54(pt 5):1469-1476.

64. Reichardt N, et al. Phylogenetic distribution of three pathways for propionate production within the human gut microbiota. ISME J. 2014;8(6):1323-1335.

65. Louis P, Young P, Holtrop G, Flint HJ. Diversity of human colonic butyrate-producing bacteria revealed by analysis of the butyryl-CoA:acetate CoA-transferase gene. Environ Microbiol. 2010;12(2):304-314.

66. Bergman EN. Energy contributions of volatile fatty acids from the gastrointestinal tract in various species. Physiol Rev. 1990;70(2):567-590.
67. Howard BV. Acetate as a carbon source for lipid synthesis in cultured cells. Biochim Biophys Acta. 1977;488(1):145-151.

68. Astakhova L, et al. Short chain fatty acids (SCFA) reprogram gene expression in human malignant epithelial and lymphoid cells. PLoS One. 2016;11(7):e0154102.

69. Nastasi C, et al. The effect of short-chain fatty acids on human monocyte-derived dendritic cells. Sci Rep. 2015;5:16148.

70. Peng L, Li ZR, Green RS, Holzman IR, Lin J. Butyrate enhances the intestinal barrier by facilitating tight junction assembly via activation of AMP-activated protein kinase in Caco-2 cell monolayers. J Nutr. 2009;139(9):1619-1625.

71. Vinolo MA, et al. SCFAs induce mouse neutrophil chemotaxis through the GPR43 receptor. PLoS One. 2011;6(6):e21205.

72. Bindels LB, et al. Gut microbiota-derived propionate reduces cancer cell proliferation in the liver. Br JCancer. 2012;107(8):1337-1344.

73. Blottière HM, Buecher B, Galmiche JP, Cherbut C. Molecular analysis of the effect of short-chain fatty acids on intestinal cell proliferation. Proc Nutr Soc. 2003;62(1):101-106

74. Siavoshian S, et al. Butyrate and trichostatin A effects on the proliferation/differentiation of human intestinal epithelial cells: induction of cyclin D3 and p21 expression. Gut. 2000;46(4):507-514.

75. Matthews GM, Howarth GS, Butler RN. Shortchain fatty acids induce apoptosis in colon cancer cells associated with changes to intracellular redox state and glucose metabolism. Chemotherapy. 2012;58(2):102-109.

76. Tang Y, Chen Y, Jiang H, Nie D. Short-chain fatty acids induced autophagy serves as an adaptive strategy for retarding mitochondria-mediated apoptotic cell death. Cell Death Differ 2011;18(4):602-618.

77. Waldecker M, Kautenburger T, Daumann $\mathrm{H}$, Busch C, Schrenk D. Inhibition of histone-deacetylase activity by short-chain fatty acids and some polyphenol metabolites formed in the colon. J Nutr Biochem. 2008;19(9):587-593.

78. Yuille S, Reichardt N, Panda S, Dunbar H, Mulde IE. Human gut bacteria as potent class I histone deacetylase inhibitors in vitro through production of butyric acid and valeric acid. PLoS One. 2018;13(7):e0201073.

79. Brown AJ, et al. The orphan G protein-coupled receptors GPR41 and GPR43 are activated by propionate and other short chain carboxylic acids. J Biol Chem . 2003;278(13):11312-11319.

80. Thangaraju M, et al. GPR109A is a G-protein-coupled receptor for the bacterial fermentation product butyrate and functions as a tumor suppressor in colon. Cancer Res. 2009;69(7):2826-2832.

81. Pluznick J. A novel SCFA receptor, the microbiota, and blood pressure regulation. Gut Microbes. 2014;5(2):202-207.

82. Gurav A, Sivaprakasam S, Bhutia YD, Boettger T, Singh N, Ganapathy V. Slc5a8, a Na+-coupled high-affinity transporter for short-chain fatty acids, is a conditional tumour suppressor in colon that protects against colitis and colon cancer under low-fibre dietary conditions. Biochem J.
2015;469(2):267-278

83. Singh N, et al. Activation of Gpr109a, receptor for niacin and the commensal metabolite butyrate, suppresses colonic inflammation and carcinogenesis. Immunity. 2014;40(1):128-139.

84. Corrêa-Oliveira R, Fachi JL, Vieira A, Sato FT, Vinolo MA. Regulation of immune cell function by short-chain fatty acids. Clin Transl Immunology. 2016;5(4):e73.

85. Donohoe DR, et al. The microbiome and butyrate regulate energy metabolism and autophagy in the mammalian colon. Cell Metab. 2011;13(5):517-526.

86. Sunkara LT, Jiang W, Zhang G. Modulation of antimicrobial host defense peptide gene expression by free fatty acids. PLoS One. 2012;7(11):e49558

87. Macia L, et al. Metabolite-sensing receptors GPR43 and GPR109A facilitate dietary fibre-induced gut homeostasis through regulation of the inflammasome. Nat Commun. 2015;6:6734.

88. Chang PV, Hao L, Offermanns S, Medzhitov R. The microbial metabolite butyrate regulates intestinal macrophage function via histone deacetylase inhibition. Proc Natl Acad Sci U S A. 2014;111(6):2247-2252.

89. Vinolo MA, Hatanaka E, Lambertucci RH, Newsholme P, Curi R. Effects of short chain fatty acids on effector mechanisms of neutrophils. Cell Biochem Funct. 2009;27(1):48-55.

90. Arpaia N, et al. Metabolites produced by commensal bacteria promote peripheral regulatory $\mathrm{T}$-cell generation. Nature. 2013;504(7480):451-455.

91. Furusawa Y, et al. Commensal microbe-derived butyrate induces the differentiation of colonic regulatory T cells. Nature. 2013;504(7480):446-450

92. Bollrath J, Powrie F. Immunology. Feed your Tregs more fiber. Science. 2013;341(6145):463-464.

93. Sakaguchi S, Yamaguchi T, Nomura T, Ono M. Regulatory T cells and immune tolerance. Cell. 2008;133(5):775-787.

94. Atarashi K, et al. Induction of colonic regulatory $T$ cells by indigenous Clostridium species. Science. 2011;331(6015):337-341.

95. Mariño E, et al. Gut microbial metabolites limit the frequency of autoimmune T cells and protect against type 1 diabetes. Nat Immunol. 2017;18(5):552-562.

96. Säemann MD, et al. Bacterial metabolite interference with maturation of human monocyte-derived dendritic cells. J Leukoc Biol. 2002;71(2):238-246

97. Iwami K, Moriyama T. Effects of short chain fatty acid, sodium butyrate, on osteoblastic cells and osteoclastic cells. Int J Biochem. 1993;25(11):1631-1635.

98. Candido EP, Reeves R, Davie JR. Sodium butyrate inhibits histone deacetylation in cultured cells. Cell. 1978;14(1):105-113.

99. Riggs MG, Whittaker RG, Neumann JR, Ingram VM. n-Butyrate causes histone modification in HeLa and Friend erythroleukaemia cells. Nature. 1977;268(5619):462-464.

100. Kim DS, et al. Attenuation of rheumatoid inflammation by sodium butyrate through reciprocal targeting of HDAC2 in osteoclasts and HDAC8 in T cells. Front Immunol. 2018;9:1525.

101. Rahman MM, Kukita A, Kukita T, Shobuike T, 
Nakamura T, Kohashi O. Two histone deacetylase inhibitors, trichostatin A and sodium butyrate, suppress differentiation into osteoclasts but not into macrophages. Blood.2003;101(9):3451-3459.

102. Nakamura T, et al. Inhibition of histone deacetylase suppresses osteoclastogenesis and bone destruction by inducing IF $\mathrm{N}-\beta$ production . JImmunol. 2005;175(9):5809-5816.

103. Cantley MD, et al. Inhibitors of histone deacetylases in class I and class II suppress human osteoclasts in vitro. JCell Physiol. 2011;226(12):3233-3241.

104.Pham L, et al. HDAC3 and HDAC7 have opposite effects on osteoclast differentiation. J Biol Chem. 2011;286(14):12056-12065.

105. Wauquier $\mathrm{F}$, et al. The free fatty acid receptor $\mathrm{G}$ protein-coupled receptor 40 (GPR40) protects from bone loss through inhibition of osteoclast differentiation. J Biol Chem. 2013;288(9):6542-6551.

106.Zaiss MM, et al. Increased bone density and resistance to ovariectomy-induced bone loss in FoxP3-transgenic mice based on impaired osteoclast differentiation. Arthritis Rheum. 2010;62(8):2328-2338.

107.Zaiss MM, et al. Treg cells suppress osteoclast formation: a new link between the immune system and bone. Arthritis Rheum. 2007;56(12):4104-4112.

108. Kelchtermans H, Geboes L, Mitera T, Huskens D, Leclercq G, Matthys P. Activated $\mathrm{CD} 4{ }^{+} \mathrm{CD} 25^{+}$ regulatory $\mathrm{T}$ cells inhibit osteoclastogenesis and collagen-induced arthritis. Ann Rheum Dis. 2009;68(5):744-750.

109. Kim YG, Lee CK, Nah SS, Mun SH, Yoo B, Moon HB. Human $\mathrm{CD} 4^{+} \mathrm{CD} 25^{+}$regulatory T cells inhibit the differentiation of osteoclasts from peripheral blood mononuclear cells. Biochem Biophys Res Commun. 2007;357(4):1046-1052.

110. Bozec A, et al. T cell costimulation molecules CD80/86 inhibit osteoclast differentiation by inducing the IDO/tryptophan pathway. Sci Transl Med. 2014;6(235):235ra60.

111. Rudnicki MA, Williams BO. Wnt signaling in bone and muscle. Bone. 2015;80:60-66.

112. Kobayashi Y, Uehara S, Udagawa N, Takahashi N. Regulation of bone metabolism by Wnt signals. J Biochem. 2016;159(4):387-392.

113. Krishnan V, Bryant HU, Macdougald OA. Regulation of bone mass by Wnt signaling. JClin Invest. 2006;116(5):1202-1209.

114. Babij P, et al. High bone mass in mice expressing a mutant LRP5 gene. J Bone Miner Res. 2003;18(6):960-974.

115. Kato M, et al. Cbfa1-independent decrease in osteoblast proliferation, osteopenia, and persistent embryonic eye vascularization in mice deficient in Lrp5, a Wnt coreceptor. J Cell Biol. 2002;157(2):303-314.

116. Guo J, et al. Suppression of Wnt signaling by Dkk1 attenuates PTH-mediated stromal cell response and new bone formation. Cell Metab. 2010;11(2):161-171.

117. Lewiecki EM. Role of sclerostin in bone and cartilage and its potential as a therapeutic target in bone diseases. Ther Adv Musculoskelet Dis. 2014;6(2):48-57.

118. Chen TH, Chen WM, Hsu KH, Kuo CD, Hung SC. Sodium butyrate activates ERK to regulate differentiation of mesenchymal stem cells. Biochem
Biophys Res Commun. 2007;355(4):913-918.

119. Katono T, et al. Sodium butyrate stimulates mineralized nodule formation and osteoprotegerin expression by human osteoblasts. Arch Oral Biol. 2008;53(10):903-909.

120. Weaver CM, et al. Galactooligosaccharides improve mineral absorption and bone properties in growing rats through gut fermentation. J Agric Food Chem. 2011;59(12):6501-6510.

121. Fujisaki J, et al. In vivo imaging of Treg cells providing immune privilege to the haematopoietic stemcell niche. Nature. 2011;474(7350):216-219.

122. Lei H, Schmidt-Bleek K, Dienelt A, Reinke P, Volk HD. Regulatory T cell-mediated anti-inflammatory effects promote successful tissue repair in both indirect and direct manners. Front Pharmacol. 2015;6:184.

123. Qiu X, Gui Y, Xu Y, Li D, Wang L. DHEA promotes osteoblast differentiation by regulating the expression of osteoblast-related genes and Foxp3(+) regulatory T cells. Biosci Trends. 2015;9(5):307-314.

124. Yu M, et al. Regulatory T cells are expanded by Teriparatide treatment in humans and mediate intermittent PTH-induced bone anabolism in mice. EMBO Rep. 2018;19(1):156-171.

125. Jilka RL. Molecular and cellular mechanisms of the anabolic effect of intermittent PTH. Bone. 2007;40(6):1434-1446.

126. Bodine PV, Komm BS. Wnt signaling and osteoblastogenesis. Rev Endocr Metab Disord. 2006;7(1-2):33-39.

127. Almeida M, Han L, Bellido T, Manolagas SC, Kousteni S. Wnt proteins prevent apoptosis of both uncommitted osteoblast progenitors and differentiated osteoblasts by $\beta$-catenin-dependent and -independent signaling cascades involving Src/ERK and phosphatidylinositol 3-kinase/ AKT. J Biol Chem. 2005;280(50):41342-41351.

128. Tobimatsu T, et al. Parathyroid hormone increases beta-catenin levels through Smad3 in mouse osteoblastic cells. Endocrinology. 2006;147(5):2583-2590.

129. Bodine PV, et al. The Wnt antagonist secreted frizzled-related protein-1 controls osteoblast and osteocyte apoptosis. JCell Biochem. 2005;96(6):1212-1230.

130. Glass DA, et al. Canonical Wnt signaling in differentiated osteoblasts controls osteoclast differentiation. Dev Cell. 2005;8(5):751-764.

131. Zmuda JM, et al. Association analysis of WNT10B with bone mass and structure among individuals of African ancestry. J Bone Miner Res. 2009;24(3):437-447.

132. Bennett CN, et al. Regulation of osteoblastogenesis and bone mass by Wnt10b. Proc Natl Acad Sci U S A. 2005;102(9):3324-3329.

133. Bennett CN, et al. Wnt10b increases postnatal bone formation by enhancing osteoblast differentiation. J Bone Miner Res. 2007;22(12):1924-1932.

134. Stevens JR, Miranda-Carboni GA, Singer MA, Brugger SM, Lyons KM, Lane TF. Wnt10b deficiency results in age-dependent loss of bone mass and progressive reduction of mesenchymal progenitor cells. J Bone Miner Res. 2010;25(10):2138-2147.

135. Zhang J, Motyl KJ, Irwin R, MacDougald OA, Britton RA, McCabe LR. Loss of bone and Wnt10b expression in male type 1 diabetic mice is blocked by the probiotic Lactobacillus reuteri. Endocrinology. 2015;156(9):3169-3182.

136. Terauchi M, et al. Tlymphocytes amplify the anabolic activity of parathyroid hormone through Wnt10b signaling. Cell Metab. 2009;10(3):229-240.

137. Bedi B, et al. Silencing of parathyroid hormone (PTH) receptor 1 in T cells blunts the bone anabolic activity of PTH. Proc Natl Acad Sci U S A. 2012;109(12):E725-E733.

138. Li JY, Walker LD, Tyagi AM, Adams J, Weitzmann MN, Pacifici R. The sclerostin-independent bone anabolic activity of intermittent PTH treatment is mediated by T-cell-produced Wnt10b. J Bone Miner Res. 2014;29(1):43-54.

139. Fehr T, et al. A CD8 T cell-intrinsic role for the calcineurin-NFAT pathway for tolerance induction in vivo. Blood. 2010;115(6):1280-1287.

140. Macián F, García-Cózar F, Im SH, Horton HF, Byrne MC, Rao A. Transcriptional mechanisms underlying lymphocyte tolerance. Cell. 2002;109(6):719-731.

141. Macian F. NFAT proteins: key regulators of T-cell development and function. Nat Rev Immunol. 2005;5(6):472-484.

142. Qin L, Raggatt LJ, Partridge NC. Parathyroid hormone: a double-edged sword for bone metabolism. Trends Endocrinol Metab. 2004;15(2):60-65.

143. Kramer I, Loots GG, Studer A, Keller H, Kneissel M. Parathyroid hormone (PTH)-induced bone gain is blunted in SOST overexpressing and deficient mice. J Bone Miner Res. 2010;25(2):178-189.

144.Feske S. Calcium signalling in lymphocyte activation and disease. Nat Rev Immunol. 2007;7(9):690-702.

145. Straub RH, Cutolo M, Pacifici R. Evolutionary medicine and bone loss in chronic inflammatory diseases - a theory of inflammation-related osteopenia. Semin Arthritis Rheum. 2015;45(2):220-228.

146.Nagpal R, et al. Human-origin probiotic cocktail increases short-chain fatty acid production via modulation of mice and human gut microbiome. Sci Rep. 2018;8(1):12649.

147. LeBlanc JG, Chain F, Martín R, Bermúdez-Humarán LG, Courau S, Langella P. Beneficial effects on host energy metabolism of short-chain fatty acids and vitamins produced by commensal and probiotic bacteria. Microb Cell Fact. 2017;16(1):79.

148. Weaver CM. Diet, gut microbiome, and bone health. Curr Osteoporos Rep. 2015;13(2):125-130.

149. Jones RM, Mercante JW, Neish AS. Reactive oxygen production induced by the gut microbiota: pharmacotherapeutic implications. Curr Med Chem. 2012;19(10):1519-1529.

150. Yan F, Polk DB. Probiotics and immune health. Curr Opin Gastroenterol. 2011;27(6):496-501.

151. Ardita CS, et al. Epithelial adhesion mediated by pilin $\mathrm{SpaC}$ is required for Lactobacillus rhamnosus GG-induced cellular responses. Appl Environ Microbiol. 2014;80(16):5068-5077.

152. Wentworth CC, Jones RM, Kwon YM, Nusrat A, Neish AS. Commensal-epithelial signaling mediated via formyl peptide receptors. Am J Pathol. 2010;177(6):2782-2790.

153. Jones RM, et al. Lactobacilli modulate epithelial cytoprotection through the Nrf2 pathway. Cell Rep. 2015;12(8):1217-1225. 
154. Alam A, et al. Redox signaling regulates commensal-mediated mucosal homeostasis and restitution and requires formyl peptide receptor 1. Mucosal Immunol. 2014;7(3):645-655.

155. Jones RM, et al. Symbiotic lactobacilli stimulate gut epithelial proliferation via Nox-mediated generation of reactive oxygen species. EMBO J. 2013;32(23):3017-3028.

156. Messora MR, et al. Probiotic therapy reduces periodontal tissue destruction and improves the intestinal morphology in rats with ligature-induced periodontitis. J Periodontol. 2013;84(12):1818-1826.

157. Mutuş R, Kocabagli N, Alp M, Acar N, Eren M, Gezen SS. The effect of dietary probiotic supplementation on tibial bone characteristics and strength in broilers. Poult Sci.2006;85(9):1621-1625.

158. Narva M, et al. Effects of long-term intervention with Lactobacillus helveticus-fermented milk on bone mineral density and bone mineral content in growing rats. Ann Nutr Metab 2004;48(4):228-234.

159. McCabe LR, Irwin R, Schaefer L, Britton RA. Probiotic use decreases intestinal inflammation and increases bone density in healthy male but not female mice. JCell Physiol. 2013;228(8):1793-1798. 160.Lei M, Hua LM, Wang DW. The effect of probiotic treatment on elderly patients with distal radius fracture: a prospective double-blind, placebo-controlled randomised clinical trial. Benef Microbes. 2016;7(5):631-637.

161. McCabe L, Britton RA, Parameswaran N. Prebiotic and probiotic regulation of bone health: role of the intestine and its microbiome. Curr Osteoporos Rep. 2015;13(6):363-371.

162.Chonan O, Matsumoto K, Watanuki M. Effect of galactooligosaccharides on calcium absorption and preventing bone loss in ovariectomized rats.
Biosci Biotechnol Biochem. 1995;59(2):236-239.

163. García-Vieyra MI, Del Real A, López MG. Agave fructans: their effect on mineral absorption and bone mineral content. JMed Food. 2014;17(11):1247-1255.

164. Abrams SA, et al. A combination of prebiotic short- and long-chain inulin-type fructans enhances calcium absorption and bone mineralization in young adolescents. Am J Clin Nutr. 2005;82(2):471-476.

165. Slevin MM, et al. Supplementation with calcium and short-chain fructo-oligosaccharides affects markers of bone turnover but not bone mineral density in postmenopausal women. J Nutr. 2014;144(3):297-304.

166. Wikoff WR, et al. Metabolomics analysis reveals large effects of gut microflora on mammalian blood metabolites. Proc Natl Acad Sci U S A. 2009;106(10):3698-3703. 